\title{
Factores determinantes de la salud y la enfermedad: mujeres en condición de desplazamiento en Medellín, Colombia 2015*
}

\section{Determinants of Health and IIIness: Women in Displacement in Medellín, Colombia 2015}

\section{Fatores determinantes da saúde e doença: Mulheres em condição de deslocamento em Medellín, Colombia 2015}

Fecha de recepción: 16-06-16 Fecha de aceptación: 06-09-16 Disponible en línea: 30-11-16 doi:10.11144/Javeriana.rgyps15-31.fdse

Cómo citar este artículo:

Posada-Zapata IC, Mendoza-Ríos A, Restrepo-Correa C, Cano-Bedoya SM, Orozco-Giraldo IC. Factores determinantes de la salud y la enfermedad: mujeres en condición de desplazamiento en Medellín, 2015. Rev. Gerenc. Polít. Salud. 2016; 15(31): 262-276. http://dx.doi.org/10.11144/Javeriana.rgyps15-31.fdse

\author{
Isabel Cristina Posada-Zapata ** \\ Abraham Mendoza-Ríos *** \\ Carolina Restrepo-Correa $* * * *$ \\ Sara María Cano-Bedoya $* * * * *$ \\ Isabel Cristina Orozco-Giraldo $* * * * * *$
}

\footnotetext{
Artículo científico de investigación. Título del proyecto: Percepción de la situación de salud/enfermedad asociada a los roles de género en mujeres en situación de desplazamiento ubicadas en los asentamientos de la Cruz, Mano de Dios y Nuevo Amanecer en la Ciudad de Medellín. 2013-2014. Fecha de inicio: 18-12-2013, fecha de finalización: 18-06-2015. El proyecto fue aprobado en la Convocatoria Programática Ciencias Sociales, Humanidades y Artes del Comité para el Desarrollo de la Investigación de la Universidad de Antioquia, 2013, mediante acta 647 del 16 de septiembre del 2013.

** Psicóloga, magíster en Salud Pública. Profesora de la Facultad Nacional de Salud Pública, Universidad de Antioquia, Medellín, Colombia. Dirección: Calle 62 \# 52-59, Facultad Nacional de Salud Pública, Of. 33-102, Universidad de Antioquia, Medellín, Colombia. Correo electrónico: isabel.posada@udea.edu.co

*** Administrador en Salud con énfasis en Gestión de Servicios de Salud, Universidad de Antioquia, Medellín, Colombia Correo electrónico: amsaludp@gmail.com

****** Estudiante de Administración en Salud con énfasis en Gestión de Servicios de Salud, Universidad de Antioquia, Medellín, Colombia. Correo electrónico: restrepocorreac@gmail.com

****** Estudiante de Administración en Salud con énfasis en Gestión de Servicios de Salud, Universidad de Antioquia, Medellín, Colombia. Correo electrónico: cano12.maria@gmail.com

******* Estudiante de Administración en Salud con énfasis en Gestión de Servicios de Salud, Universidad de Antioquia, Medellín, Colombia. Correo electrónico: isabelorozcogiraldo@gmail.com
} 


\section{Resumen}

Desde un enfoque de determinantes sociales, se ha demostrado la relación existente entre las condiciones de vida estructurales y la situación de salud o enfermedad de poblaciones específicas. Esta relación tiende a complejizarse cuando se viven situaciones de conflicto, las cuales agudizan la vulnerabilidad. El objetivo fue describir las vivencias de mujeres en condición de desplazamiento y sus efectos en los procesos de salud-enfermedad. Con un enfoque cualitativo, se tomaron aspectos de la teoría fundamentada de Strauss y Corbin. Participaron 49 mujeres en situación de desplazamiento. Para el análisis se utilizó la codificación, la categorización y la interpretación, y finalmente se llevó a cabo la teorización. Se consideró la descripción conceptual de percepción, significado, la salud-enfermedad, la salud pública, el género y el desplazamiento. Como hallazgo principal se encontró que el desplazamiento y las representaciones socioculturales marcan considerablemente la significación y la forma de asumir los procesos de salud-enfermedad de las mujeres.

Palavras-chave: migración; salud; enfermedad; salud de la mujer; rol; actividades cotidianas; determinantes sociales de la salud

\section{Abstract}

The relationship between structural life conditions and the health status of specific populations has been proven through a social determinants approach. Moreover, this relationship tends to become more complex with the appearance of conflicts that accentuate vulnerability. The aim of this study was to describe the life experiences of women displaced by violence and the effects of displacement on health/disease processes. A qualitative approach with elements from Strauss' and Corbin's Grounded Theory was used in the study, which included a total of 49 displaced women. Data were analyzed via coding, categorization and interpretation before theorizing. In addition, the conceptual description of perception, meaning, health/disease, public health, gender and displacement was taken into account. The main finding was that displacement and social and cultural representations have a considerable effect on the meaning and manner in which the women's health/disease processes are perceived.

Keywords: migration; health; disease; women's health; role; activities of daily living; social determinants of health

\section{Resumo}

A partir de um foco dos determinantes sociais, demostrou-se a relação existente entre as condições de vida estruturais e a situação de saúde ou doença de populações especificas. Esse relacionamento tende a complexificar-se quando são vividas situações de conflito, as quais exacerbam a vulnerabilidade. O objetivo foi descrever as experiências de mulheres em condição de deslocamento e seus efeitos nos processos de saúde-doença. Com um foco qualitativo, foram tomados aspectos da teoria fundamentada de Strauss e Corbin. Participaram 49 mulheres em situação de deslocamento. Para o análise foi utilizada a codificação, a categorização e interpretação, e finalmente foi realizada a teorização. Foi considerado a descrição conceitual de percepção, significado, a saúde-doença, a saúde pública, o gênero e o deslocamento. O principal resultado foi que o deslocamento e as representações socioculturais marcam consideravelmente a significação e o jeito de levar os processos de saúde-doença das mulheres.

Palabras clave: migração; saúde; doença; saúde da mulher; papel; atividades cotidianas; determinantes sociais da saúde 


\section{Introducción}

En Colombia, el conflicto armado ha ocasionado una grave crisis a nivel social, económico, político y sobre todo humanitario. Este fenómeno ha generado serias vulneraciones a los derechos humanos, fundamentalmente a la vida, la libertad, la integridad física, los derechos individuales y políticos, los económicos, sociales y culturales. Las consecuencias de este conflicto han afectado principalmente a la población civil, la cual ha sido víctima de desplazamiento forzoso, homicidios ilegítimos, minas antipersona, torturas, desaparición forzosa o secuestro y abusos sexuales a manos de todas las partes enfrentadas $(1,2)$.

De acuerdo con el Registro Único de Víctimas, Colombia ocupa el segundo lugar en el mundo en número de desplazados internos, antecedido por Siria. Actualmente, en Colombia hay 5.7 millones de personas desplazadas, lo que supone que 1 de cada 10 personas en Colombia se encuentra en esta condición (3).

En el marco del conflicto armado, los grupos sociales más vulnerables se ven altamente afectados. Entre ellos se encuentran las mujeres, quienes en el caso de Colombia representan el $51.2 \%$ del total de las víctimas, y enfrentan violaciones contra la libertad y la integridad sexual, ya que violentar el cuerpo femenino es empleado como táctica de guerra, y los abusos contra los derechos humanos de las mujeres forman parte de las estrategias bélicas. En efecto, en Colombia la violencia sexual se considera una práctica sistemática, habitual y generalizada (4).

Como consecuencia de la ola de violencia, Medellín ha sido receptora de familias completas que han huido de los enfrentamientos y amenazas de las fuerzas armadas oficiales 264 e insurgentes. Dado que la ciudad no dejó nunca de recibir población en busca de segu- ridad para sus familias, las laderas se fueron poblando densamente y en los asentamientos se constituyeron comunidades que rápidamente iniciaron un proceso de organización, lucha por sus derechos y por la inclusión social como ciudadanos.

Según Hernández Bello y Gutiérrez Bonilla, "el desplazamiento constituye un proceso de desarraigo, de ruptura de las redes sociales y lazos culturales, de pérdidas en bienestar, bienes patrimoniales y medios de subsistencia, miedo e incertidumbre que hacen de los desplazados poblaciones vulnerables", hecho que obliga a las mujeres víctimas de este acontecimiento a realizar una reestructuración de sus condiciones de vida en la ciudad receptora, de adaptación a unas dinámicas sociales, políticas y económicas que son diferentes a las de sus lugares de origen, y a una búsqueda de soluciones que aporten al desarrollo individual y familiar y permitan la dignificación de sus vidas. Sin embargo, estos procesos se dificultan debido a que la ciudad receptora no es garante de condiciones de vida adaptadas a las necesidades de esta población, y las mujeres desplazadas se ven enfrentadas a factores como el empleo informal, bajos recursos económicos, necesidades básicas insatisfechas y escasez alimentaria y nutricional (5).

En ese mismo contexto, las mujeres desplazadas asumen diferentes y nuevas responsabilidades, lo que trae consigo grandes crisis y problemas en la salud física y mental que difícilmente pueden cuantificarse. En efecto, su salud se ve altamente comprometida, lo que se agudiza porque el acceso a los servicios de salud en la ciudad receptora es bastante complejo y discriminatorio (6).

Es por ello que los nuevos roles que desempeñan las mujeres en situación de desplazamiento, las experiencias vividas antes, durante y después de este acontecimiento, y las condiciones de vida en la ciudad re- 
ceptora, impactan en su calidad de vida, lo que determina la manera como asumen los procesos de salud-enfermedad a partir de dichas vivencias. Es con base en estas experiencias que se ha definido particularmente tanto el concepto de salud como el de enfermedad, delimitados por la subjetividad de pensamientos y acciones en un contexto familiar, individual y colectivo que influye directamente en la percepción del estado de salud de estas mujeres.

De allí la importancia de preguntarse cuál es la percepción que ellas tienen con respecto a su situación de salud-enfermedad, a partir de su condición de mujeres desplazadas y de factores con los que interactúan en su vida cotidiana. Responder esta pregunta dará luces a las acciones que se encaminen a mejorar la salud integral de este grupo poblacional, de acuerdo con sus necesidades específicas, y, de esta manera, redundar en el bienestar de sus hijos, familias y contextos cercanos.

En ese sentido, el proyecto pretende un abordaje desde la percepción y las vivencias de las mujeres de tres asentamientos de la ciudad de Medellín, para que en el futuro y con las herramientas de la salud pública y la salud mental, y desde un enfoque de salud sexual y reproductiva, se puedan construir soluciones de forma conjunta y participativa.

Este artículo tiene como objetivo describir las vivencias de las mujeres en condición de desplazamiento y cómo dichas vivencias afectan los procesos de salud-enfermedad.

\section{Materiales y métodos}

Esta investigación se llevó a cabo de acuerdo con un enfoque cualitativo y holístico, ya que se buscó analizar e interpretar de la forma más amplia posible los diferentes significados de las vivencias de las mujeres desplazadas con relación a la percepción de su estado de saludenfermedad. Con apoyo en el interaccionismo simbólico, se determinaron los significados de los actos y las palabras de este grupo de personas y la forma como se construyen en la interacción con otros, para finalmente agruparlos y constituir un conjunto de sentidos alrededor del discurso de estas mujeres (7).

De esta manera, se utilizó la teoría fundada como método de investigación, entendida como un modelo explicativo derivado de datos recopilados sistemáticamente y analizados por medio de un proceso de investigación. En este método, la recolección de datos, el análisis y la teoría que surgirá de ellos guardan estrecha relación entre sî (7). Consecuentemente, se buscó hacer una interpretación de los conceptos ligados a las experiencias de estas mujeres y se desarrolló una categorización de los datos.

Los criterios de inclusión de la población fueron ser mujer mayor de edad, vivir en condición de desplazamiento durante al menos un año y residir en alguno de los tres asentamientos seleccionados. Se realizaron quince entrevistas iniciales (E), seis grupos focales (GF) y seis entrevistas a profundidad (EP), con una participación total de 49 mujeres.

Estas mujeres fueron contactadas en tres asentamientos en Medellín. El asentamiento La Cruz está ubicado al extremo nororiental de la ciudad y hace parte de la comuna 3 (Manrique); allí la población en su mayoría está formada por desplazados. Sus condiciones sociales son de alta vulnerabilidad - viviendas, acceso limitado a servicios públicos, educación, salud, alimentación, movilidad y pocas vías de acceso- - y sus ocupaciones laborales se concentran en la construcción, los oficios varios y la informalidad. El desempleo también es una constante, lo que dificulta contrarrestar las problemáticas sociales que se presentan (8). 
Mano de Dios era un asentamiento que se encontraba cerca del centro de Medellín y también era receptora de familias desplazadas, en su mayoría afrodescendientes que migraron de sus lugares de origen por el conflicto armado. En el año 2003 en este asentamiento hubo un incendio que dejó muchos damnificados y como consecuencia estas familias sufrieron un desplazamiento interno en la ciudad, lo que significó un doble desplazamiento para aquellos que ya habían pasado por esta experiencia. Estas personas fueron reubicadas en el barrio Nuevo Amanecer, en el corregimiento Belén Altavista, conformado por 3600 personas aproximadamente, quienes asimismo viven en condiciones sociales precarias como, por ejemplo, contaminación ambiental a causa de la cercanía del barrio a la industria ladrillera del sector, escasas oportunidades de empleo, acceso limitado a la salud, discriminación y violencia.

Se tuvieron en cuenta dos tipos de fuentes de información: primarias, constituidas por los discursos de las participantes; y secundarias, constituidas por la revisión de documentos oficiales, literatura y otras investigaciones.

En las entrevistas iniciales se trataron temas como la experiencia del desplazamiento, la relación entre los roles que desempeña la mujer con su estado de salud y la percepción de su familia. En los grupos focales se habló de cómo las mujeres aportaban al cuidado de su salud y de sus familias, cómo había cambiado el cuidado de su salud luego del desplazamiento y en la relación de las condiciones sociales con la enfermedad. Finalmente, las entrevistas a profundidad se realizaron con el fin de poner a prueba las hipótesis que se habían construido durante toda la investigación. zó el muestreo teórico, lo que implicó elegir la población de acuerdo con las necesidades de las categorías referencia; es decir, inicialmente, las mujeres fueron seleccionadas con el fin de obtener un grupo con características sociodemográficas diferentes que aportará información desde varias perspectivas. En las otras fases de recolección de información se eligió a aquellas mujeres que, a partir de los resultados obtenidos, consiguieran alimentar la investigación con base en el conocimiento de la comunidad o liderazgo.

El plan de análisis consistió en tres procedimientos básicos: la codificación, a través de un análisis línea por línea de las entrevistas realizadas con las participantes, iniciando con una codificación abierta, pasando a una analítica que busca relaciones y, finalmente, llegando a una selectiva que busca fortalecer la interpretación; la categorización, donde se realizó una agrupación posterior en conjuntos que respondían desde lo descriptivo, lo analítico y lo interpretativo; y por último se llevó a cabo la teorización, es decir, la construcción de un modelo explicativo que respondiera a la pregunta de investigación (7).

Luego del análisis, se realizó la validación de los hallazgos por medio de una socialización ante la comunidad en general de los asentamientos, donde las participantes reconocieron la validez de los resultados expuestos.

Para la ejecución de esta investigación se tuvieron en cuenta los criterios establecidos en la Declaración de Helsinki, donde se insta a los investigadores a garantizar la protección de la vida, la salud, la dignidad, la integridad, la libre determinación, la privacidad y la confidencialidad de la información personal de la población sujeto (9). Así mismo, se tuvo en cuenta la Resolución 8430 de 1993 (10) del Ministerio de Salud de Colombia, que regula la investigación con seres humanos, y desde allí este proyecto fue catalogado como de riesgo mayor al mínimo, por lo que siempre 
se estuvo atento a los posibles riesgos psicosociales que se derivaran del discurso de las participantes, y se aplicó además el formato de consentimiento informado. Teniendo en cuenta estas consideraciones, se obtuvo el aval del Comité de Ética de la Investigación de la Facultad Nacional de Salud Pública de la Universidad de Antioquia.

\section{Hallazgos}

Las mujeres en situación de desplazamiento viven de maneras diferentes y construyen culturalmente el significado de salud, desde una representación biopsicosocial hasta una perspectiva política donde la salud es vista como un derecho fundamental, tomando en cuenta determinantes sociales, culturales, económicos y ambientales que influyen en la significancia del concepto. Es por ello que la salud puede consistir en el hecho mismo de tener salud, de estar saludable; entonces, su definición se multiplica infinitamente, ya que la vivencia de estar sano es diferente en cada persona (11).

$\mathrm{Al}$ tomar esto como referencia para las mujeres desplazadas, el concepto de salud se relaciona con un estado de bienestar integral que abarca no solo lo físico, sino también lo psicológico y lo espiritual, descrito así por algunas de las participantes:

Pues hay diferencias en identificaciones de salud, salud física, mental, pero más que todo siempre lo generalizamos en bienestar. (E14C3)

El concepto de salud visto desde una perspectiva integradora de lo físico y lo psicológico por las mujeres, evidencia la importancia de la existencia de un equilibrio entre ambos factores para una vivencia óptima de la salud, lo que trae como consecuencia la percepción de estar saludable o enfermo.
Sin embargo, ante este equilibrio se encuentra una brecha entre cómo se comprende el concepto de salud y cómo es la vivencia de este, dado que dentro de la cotidianidad de las mujeres se encuentran determinantes sociales de la salud, entendidos como las características sociales dentro de las cuales tiene lugar la vida (12), que afectan los diferentes procesos de salud y enfermedad por los que atraviesan las mujeres en las diferentes etapas de su vida:

Es una salud buena, sobre todo también la comida, una buena comida, una buena casa, una buena dormida, todo eso es salud para mí. (E6C2)

Luego del desplazamiento las mujeres deben adaptarse a las condiciones de vida que ofrece la ciudad a la que llegan, y tienen que asumir las barreras que se les presentan a ellas y a sus familias, desde lo económico, lo laboral, lo educativo, lo social, así como las diferencias culturales, donde estos determinantes intervienen en la concepción de la salud. Las mujeres son forzadas a vivir la salud dentro de estos límites impuestos indirectamente y ello no favorece la percepción de su estado de salud.

Lograr que mejoren las condiciones de vida de las mujeres en situación de desplazamiento no depende únicamente de ellas mismas, puesto que también se ve implicado el papel del Estado como auxiliador ante estas circunstancias adversas, y de este se espera un conjunto de intervenciones que impacten de manera positiva en su bienestar, incluido el ámbito de la salud, pues es considerado "un derecho humano fundamental" (13). Así lo señala una de las entrevistadas:

[...] es como [...] pues mi concepto es algo que todos tenemos derecho ino?, es como un beneficio icierto?, un beneficio la salud 
[...] si, un beneficio que todos tenemos derecho. (E1C1)

Es el Estado el que debe comprometerse para que las mujeres en situación de desplazamiento sean vistas como ciudadanas con derechos, así como cumplir con la provisión de condiciones de vida más favorables que fomenten la salud y un óptimo desarrollo, teniendo en cuenta que si se ven alteradas las condiciones ambientales, económicas, culturales y sociales dadas en un contexto colectivo e individual en la mujer desplazada, esto se verá reflejado en su estado de salud significativamente.

En complemento de lo anterior, la vivencia del desplazamiento es un acontecimiento que impacta de manera notable a las mujeres que han sufrido dicha experiencia, y cambia la percepción de su estado de salud, debido a que en el ámbito cultural este hecho perjudica el modo de vivir; en consecuencia, las mujeres desplazadas deben reconstruirse en el nuevo contexto, cambiando sus hábitos y costumbres.

Esto conlleva que no puedan ejercer sus prácticas culturales o que no puedan desarrollarlas adecuadamente en el nuevo contexto, en vista de que no tienen las herramientas para realizarlas, el entorno en el que ellas se desenvolvían no es el mismo y las normas sociales son diferentes, lo cual limita las acciones culturales que ayudan a la mujer en la representación que tiene de su estado de salud, puesto que tanto las creencias como las prácticas del cuidado de la salud se originan y se desarrollan dentro de un contexto social, se influyen mutuamente y son parte del carácter dinámico que permite a la cultura favorecer la adaptación de la persona a su entorno. Tener en cuenta los aspectos culturales le da un toque humano al cuidado y favorece el que se alcancen los objetivos terapéuticos (14):
Sobre todo uno aquí pasa mucho trabajo porque las hierbas del Chocó no están acá. Pero una en el Chocó sí, porque uno se baña, que se coge el matarratón, que coge la verbena, que coge la sal de frutas, que... ¿Cuál otra? La esparilla, todo eso uno lo machaca y lo revuelve, el zumo del coco, que la piña $[\ldots](\mathrm{E} 6 \mathrm{C} 25)$

De la misma manera, la noción de enfermedad ha sufrido importantes transformaciones a través de la historia. Por esta razón, el ser humano siempre se ha visto en la necesidad de interpretar la enfermedad de acuerdo con el paradigma dominante (15) y las condiciones de vida que se presentan durante la historia de la sociedad, ya que la forma de definir este fenómeno queda supeditada a múltiples factores del entorno que intervienen en la percepción del individuo y determinan la manera como se viven las situaciones en las que el estado de salud se ve afectado. De este modo, la connotación de proceso de enfermedad se convierte en un elemento subjetivo, basado en las propias experiencias y vivencias de cada ser humano. En este sentido, una de las participantes expresa:

$[\ldots]$ porque es que uno se enferma mucho es de pensar, de pensar la situación económica, por ejemplo, ahora que los muchachos están en vacaciones iay Dios mío bendito! Eso es cada momentico: mamá tengo hambre, mamá compra una bolsita de leche, ay mamá yo tengo unas ganas de una gaseosa y uno sin con que comprarla entonces me da como vea, la cabeza se me va creciendo y me pongo a pensar: no, es que, que yo tuviera al menos un empleo, que yo supiera que ganaba mi platica segurita y que tengo tiempo de estar con estos pelaos, fuera muy bueno, pero iahhg! Eso sí, ayuda mucho porque uno ni come, por ejemplo, yo en mi casa si yo tengo una libra de arroz y tres huevos, yo se la hago a los niños y les doy los huevos a los niños y ya, para mí no alcanza entonces sí, se va 
poniendo uno débil. Yo los veo a ellos y yo: iqué pecao! y yo al rato soy, trague saliva y yo: iay verdad que es que yo no he comido hoy! [...] (EP5C100-103)

El concepto de enfermedad generalmente se encuentra relacionado con el hecho de presentar una sintomatología física que causa un malestar, pero en esencia es dinámico y no corresponde solo a la afectación biológica o a una simple referencia de carácter fisiológico, ya que está implicado históricamente y unido a condicionamientos culturales, sociales y económicos (16).

En el desarrollo de esta investigación se halla una relación del concepto de enfermedad con molestias físicas y padecimientos mentales de las mujeres. Dentro de los factores físicos se evidencia la influencia del entorno y condiciones de vida sobre el cuerpo que causan un malestar definido como: "no estar a gusto con ciertas cosas que manifiesta el cuerpo (E1C5)", tanto crónicas como leves, que tienen repercusiones importantes sobre el estado de salud y el desempeño de los roles de las mujeres en su cotidianidad. En las dolencias psicológicas se encuentra que el concepto de enfermedad está estrechamente relacionado con la salud mental de las mujeres, ya que se convierte en un desencadenante de problemas reflejados en la salud y en el cuerpo. Una de las entrevistas se refiere a esa conexión entre la falta de bienestar mental y el concepto de enfermedad:

Pues $[\ldots]$ primero que todo también va el estrés, la pensadera [...] y los problemas que uno vea como entre familia, también eso lo enferma a uno. (E3C4)

De esta forma, se demuestra que malestares psicológicos como la depresión, el estrés, los trastornos del estado de ánimo, los sentimientos de aflicción por situaciones familiares negativas, la ansiedad con respecto a problemas económicos, la ausencia de seres queridos por muerte o abandono, la falta de estabilidad emocional y la desesperanza repercuten, no solo en la vivencia y percepción de su estado de salud, sino también en el cumplimiento habitual de los roles que desempeñan dentro de la familia, la comunidad, el trabajo y en su papel de mujeres.

En ese contexto, el significado de la enfermedad que tienen las mujeres está ligado igualmente a la incapacidad de realizar las tareas que efectúan cotidianamente, ya que el estar enfermas representa un estado de inactividad, debido a la presencia de una limitación para ejecutarlas. Ledón Llanes afirma que es común encontrar, como resultado de los múltiples cambios físicos y psicológicos asociados al proceso de enfermedad, cambios en el desempeño de las labores. Este es uno de los aspectos que mayor distrés generan, pues conduce a los individuos a reestructurar su vida cotidiana; a veces, durante un tiempo considerable, dedicarse solo al cuidado de la propia salud, aprender a desenvolverse en nuevos espacios sociales con nuevas normas y exigencias (como los espacios de salud), y quizás también a orientar las capacidades, potencialidades y la búsqueda de autonomía en nuevas direcciones (17):

\footnotetext{
Bueno eso si me afectó [...] al principio me afectó mucho, sobre todo que no podía caminar, me tenían mis hijas que cargar, bajarme a bañar, subirme, inclusive que me tenían hasta que vestir, porque no me podía parar bien y ahí sentadita me traían la ropa y yo me vestía así. (E6C13)
}

Esta asociación entre el concepto de enfermedad en las mujeres y la limitación del ejercicio de las actividades cotidianas, se encontró con frecuencia en la preocupación que manifiestan cuando tienen dificultades en el momento de la realización de actividades domésticas, laborales o del cuidado de la 
familia; tareas que hacen parte de los roles que cumplen culturalmente:

Cuando uno se enferma o algo, entonces le toca como dejar $[\ldots]$ como $[\ldots]$ no estar tan pendiente de la niña, ya le toca a mi mamá [...] mi abuela, ella me ayuda mucho con ella, entonces si estoy enferma están pendientes de la niña, que necesita la niña, entonces antes es como un apoyo [...] (E1C47)

Una de las dimensiones más importantes de la calidad de vida es el sentido de autonomía de la persona, lo cual está muy relacionado con la posibilidad de desarrollar sus actividades cotidianas (17). La pérdida de autonomía, que para las mujeres implica recibir ayuda —en muchos casos de su familiapara el desarrollo de actividades de la vida diaria, hace parte de esa concepción global de enfermedad y la visión que repercute en ellas sobre el sentirse sanas o enfermas, como lo describe una de las participantes:

Estando enferma ya uno lo que piensa es eso y que estén los familiares de uno, dándole los primeros auxilios digamos, pues la comidita, que el baño, que llevándolo a un puesto, que levantándolo de ahí y llevándolo a la cama y todo eso. (E6C7)

Los sujetos suelen referirse a la familia como la fuente de apoyo (material, pero sobre todo emocional) por excelencia. Las relaciones interpersonales de las mujeres con la familia, la pareja y las personas cercanas a ellas constituyen las dinámicas de interacción fundamentales donde los procesos de salud y enfermedad se presentan:

\section{[...] estar enfermo es muy triste porque ima-} gínese uno acostado, uno en una cama, que no lo visiten, que uno cuando está enfermo lo tienen como aislado, y así sucesivamente, estar enfermo es muy maluco [...] (E8C11)
Por último, la mujer desplazada asocia la presencia de enfermedades con un deterioro de la funcionalidad diaria en el hogar y en su vida personal. En palabras de una de las participantes:

Me afecta porque yo no puedo, ahora que estoy estudiando, yo no puedo hacer educación física, porque de nada estoy agitada, que como me subo un poquito de peso, subo las escalas de la casa y sé que ya no puedo, o sea por cualquier cosita estoy que ya, no es como uno vivir tranquilo, que por cualquier bobada uno normal corre, salta, camina por todos los lados, y uno no siente nada, isi me entiende? pero en estos momentos como así estoy, me da mucha dificultad y por muchas cosas. (E15C7)

Esto demuestra cómo esa vivencia individual en el proceso de enfermedad interviene de forma directa en la ejecución de los roles que desempeñan las mujeres diariamente y la importancia de estos para la continua realización de sus proyectos de vida.

El trabajo es un aspecto fundamental de la vida de las personas y constituye un pilar para la estabilidad de las familias y las sociedades. Toda persona aspira a tener un trabajo que le proporcione un nivel de vida aceptable tanto para ella como para su familia; un trabajo en el que sean considerados sus derechos y opiniones (18):

Bueno, decir que uno se gana un salario mínimo, voy a cubrir todas las necesidades, eso es mentira, porque gracias a Dios isí! Yo me gano un salario mínimo, según el Estado yo tengo un trabajo digno, dentro de lo que cabe icierto?, yo soy madre cabeza de familia, velo por dos hijos que tengo, pero yo velo por mis papás que tienen más de sesenta años, yo llevo la obligación de ellos, por la alimentación de ellos, entonces aparte de ese salario mínimo, que no me alcanza, 
yo hago todos los domingos galletas y me voy por ahí a vender las galletas para cubrir otras necesidades que yo tengo porque no me alcanza. (GF1C71)

Las oportunidades de empleo para las mujeres se restringen a labores del hogar o a las ventas ambulantes en condiciones inestables, caracterizadas por una baja remuneración que resulta insuficiente para el sostenimiento del núcleo familiar. Aun cuando la población desplazada logra encontrar empleo, este se caracteriza por requerir pocas habilidades y un alto grado de informalidad; en consecuencia, genera ingresos bajos e inestables [...] (19):

\section{Sí, estaba trabajando también [...] yo estaba en otra parte pero no tenía [...] ni seguro, nada pero ganaba allá [...] ganaba veintidós mil, tocaba madrugar también mucho, de cinco a cinco de la tarde. (E1C82)}

En ese sentido, las condiciones laborales determinan la percepción del estado de salud que tienen las mujeres, debido a una carga laboral fuerte en donde se ven expuestas a largas jornadas de trabajo, sin descansos adecuados, falta de garantías laborales, múltiples funciones, en muchas ocasiones desprendimiento familiar por varios días y baja remuneración; por lo tanto, las mujeres desplazadas ven deteriorada su salud y esta situación no les permite alcanzar un óptimo estado de salud integral. Al verse afectadas por estas condiciones laborales, sienten que no son productivas, y esta puede ser la causa que desencadene, además de dolencias físicas, dolencias psicológicas donde se perciban enfermas a sí mismas:

Por ejemplo, yo cuando tuve el niño mío, yo a los seis meses me fui a trabajar interna, ehh [...] llegaba los sábados a las ocho de la noche, me tocaba entrar los domingos a la cinco de la tarde, la verdad el niño [...] cuando él creció yo no me acuerdo de las cositas de él pues como cuando empezó a gatear, todas esas cosas porque yo no tuve el tiempo para estar con él. Yo duré cuatro años trabajando interna y el niño, imagínese, en esos cuatro años pues pasan muchas cosas y yo a veces me pongo a pensar: ahh y ¿Cuándo empezó el niño a caminar? Mi abuela me lo cuidaba, él ya le estaba diciendo mamá a mi abuela, ehh [...] y él creció ya al lado de ella, él ya no se amañaba conmigo sino que era ella, la abuela. (GF6193-194)

El concepto de enfermedad está ligado a varios factores de vivencias personales que determinan la percepción del estado de salud de cada una de las mujeres. Tiene en cuenta los arraigos culturales, el significado del autocuidado y la interpretación de las alteraciones físicas y psicológicas, que en unos casos pueden llevar a la búsqueda de soluciones para maximizar el bienestar propio y el de su familia, en tanto que en otros originan pensamientos y reflexiones sobre la muerte o la improductividad.

Asimismo, la práctica o creencia de la religión hace parte de esas acciones realizadas por la mayoría de las mujeres para aumentar su bienestar mental, en vista de que la religiosidad desempeña un papel muy importante de interacción entre las mujeres y la forma en como viven los procesos de salud-enfermedad; además, permite afrontar otros aspectos de su vida, porque se refugian en ese sentir y en esas ideologías para obtener mayor fortaleza o resistencia ante las dificultades, lo que influye en la percepción que tienen de su estado de salud. Como menciona una participante:

Dios ayuda mucho, nos da fortaleza, entonces [...] después de que uno tenga fe todo es posible, mi creencia se ha $[\ldots]$ yo creo que ahora es lo que me tiene más fuerte $[\ldots]$ yo le pido mucho a Dios que $[\ldots]$ ayude en $\mathrm{mi}$ 
casa, que me ayude a ser [...] mejor madre, mejor hija. (E1C94-95)

El término religión se utiliza para referirse a un sistema organizado de creencias, prácticas, rituales y símbolos diseñados para facilitar el acercamiento a una realidad sagrada y fomentar la comprensión de la conexión y la responsabilidad de una persona a una comunidad (20).

Enfrentado a múltiples rupturas y urgentes transformaciones, el desplazado por la violencia acude a la religiosidad para justificar su presente, vincularse a los nuevos espacios o hallar referentes ciertos o conocidos (20). Por esta razón, las mujeres se refugian en la religión para lograr sus metas y proyecciones de vida actuales, a través de sentimientos de esperanza y sobrellevando las consecuencias que la experiencia del desplazamiento sembró en sus vidas particulares y las de sus familias:

Fuerte, pues porque uno tiene que seguir adelante y pedirle a Dios que, y ahora que soy jefe de hogar, seguir fuerte, Dios dice ayúdate y yo te ayudaré (se ríe), entonces uno tiene que seguir adelante, nada de pa' atrás, todo positivo, para mí no hay nada negativo. (E10C46)

De igual manera, para la mayoría de las mujeres la presencia de un Dios es tomada como "un todo" que le da sentido a la vida, como quien tiene la responsabilidad de definir cada suceso que ocurra en ella, desde su estado de salud hasta incluso la vida o la muerte:

¿Qué piensa? Pues nada ya mi mamá dice que sea la voluntad de mi Dios, porque la única solución que yo esté mejor que ahorita es que me hagan un trasplante de corazón, pero igual eso vale, cuesta mucho, mucha plata. (E15C9)
Los métodos de afrontamiento religioso también se han notificado por su efecto positivo en el bienestar físico y la salud mental de las personas mayores. Koenig, McCullough y Larson sostienen que las personas religiosas tienden a disfrutar de una mejor salud física y mental que quienes no lo son. Esto puede deberse en parte a prácticas religiosas como la meditación y la oración, que inducen lo que se conoce como una "respuesta de relajación”. Estos métodos de afrontamiento han sido empleados por las personas mayores para hacer frente al estrés, la enfermedad, la invalidez, la muerte, la pérdida, el aislamiento social y el impacto que su cambio de rol en la sociedad puede tener en sus vidas (21):

Por lo menos en mi caso [...] me siento como bien. Yo digo que me da la fortaleza que a veces como que necesito, ehh [...] me siento como bien, por ejemplo si estoy aburrida me pongo a leer algún libro de él, voy y busco en la Biblia. (E9C71)

Con base en lo relatado, se puede concluir que la manera de asumir los procesos de salud-enfermedad está determinada por diversos modos de actuar y de pensar, construidos por una representación sociocultural construida por las mujeres en situación de desplazamiento a través de los años, que posibilita adicionar a sus vidas un cúmulo de significaciones que, sumado a los determinantes sociales de la salud con los que interactúan durante el desempeño de todos sus roles, permiten generar su propia percepción del estado de salud.

\section{Discusión}

Desde hace más de cinco décadas, en Colombia el conflicto armado es un problema de salud pública que genera distintas formas de violencia y secuelas imborrables en la población (22). Según la Consultoría para los 
Derechos Humanos y el Desplazamiento, en el 2011 Colombia presentaba el índice más alto de desplazados internos y refugiados en el mundo, y entre 1985 y el 2010, la cifra acumulada de personas desplazadas de sus hogares a causa del conflicto armado, tanto fuera como dentro del país, era de 5195620 personas (19).

La génesis de esta problemática radica en multiplicidad de causas de tipo político, económico, social y cultural. Durante y después del conflicto, las mujeres se interrelacionan con otros grupos humanos y, en consecuencia, con diferentes culturas y estilos de vida. Esto implica la vivencia de circunstancias sociales como la integración, la asimilación o la segregación. Sánchez y Jaramillo plantean que en nuestro país la gran mayoría de los desplazados sufren el proceso de segregación, algunos son asimilados por la nueva cultura y solo una pequeña minoría, en situaciones muy particulares, logra el proceso de integración. Es por esto que el fenómeno del desplazamiento no es tan solo un asunto de orden jurídico o político, sino que afecta de manera vital y altera la relación de las mujeres con los procesos de salud-enfermedad. Esta relación se ve influida además por las características individuales de cada una, su percepción del acontecimiento, características psicológicas, entre otros rasgos personales y sociales (23).

García, por su parte, hace referencia a las consecuencias psicosociales en la población desplazada, e incluye la desestructuración familiar como uno de los rasgos más representativos (24). En este sentido, las mujeres resaltan que los procesos de acompañamiento, comunicación, búsqueda activa de soluciones y provisión de cuidados por parte de la familia, se convierten en estímulos fundamentales y factores clave al momento de presentar enfermedades, y favorecen la recuperación de la salud (17).
Por otro lado, a causa del desplazamiento experimentado por las mujeres y las condiciones de vida en la ciudad receptora, los determinantes sociales se presentan muchas veces de manera desfavorecedora. Así se evidenció en una entrevista realizada por Hernández y Gutiérrez donde se relata: "la alimentación, factor esencial del bienestar, se ve fuertemente afectada por la dificultad de acceder a ingresos mínimos necesarios, y hace de este componente uno de los más crueles indicadores de carencia de los hogares en general y de los hogares de los desplazados en particular” (25).

A partir del desplazamiento las mujeres y sus familias deben asumir el cubrimiento de necesidades básicas, utilizando diversas estrategias que van desde recurrir a familiares y amigos hasta buscar opciones laborales diferentes a las habituales en sus zonas de origen. La angustia constante por la seguridad propia y de sus familias y la escasez de recursos de alimentación, vivienda y calidad de vida económica genera afectaciones a la salud física y mental, entre las cuales se destacan la pérdida del sueño, baja en el peso, déficit en el comportamiento de autocuidado e incluso ideas suicidas. También el autoconcepto de las mujeres se afecta por la incapacidad percibida para autosostenerse como lo hacían habitualmente en sus zonas de origen: "El trabajo es el que proporciona el mejor soporte social, ello implica que las personas al quedar desempleadas, como consecuencia del desplazamiento, no pueden asumir un rol laboral adecuado que les permita lograr un apropiado nivel de soporte social" (23).

Es así como el derecho a la salud está estrechamente ligado al desarrollo humano y a la calidad de vida, conforme a lo explicado por la Organización Mundial de la Salud:

[...] los Estados deberían también adoptar medidas para la realización de su derecho, 
entre otras cosas, a una vivienda adecuada, a condiciones de trabajo seguras y salubres, a un nivel de vida adecuado, a la alimentación, a la información, a la libertad y la seguridad de la persona, a un proceso con todas las garantías y a no ser sometidos a la esclavitud y a trabajo forzado. (26)

La situación de desplazamiento lleva a las mujeres y sus familias a vivenciar de múltiples maneras la exclusión social y el deterioro de la calidad de vida. La alta morbilidad en salud mental se explica por la asociación entre bienestar emocional y adversidades relacionadas con los diferentes tipos de violencia que sufren $y$, por otra parte, por las desavenencias propias de la situación de desplazamiento (22).

Entre las variables que influyen en el estado de salud mental posterior a un proceso de desplazamiento se encuentran la presión por aculturarse, la falta de oportunidades económicas en el nuevo ambiente, la heterogeneidad en la cultura y el cumplimiento de expectativas y aspiraciones (23).

Además, el conflicto armado se percibe, más allá de un enfrentamiento de grupos armados, como el resultado de la falta de compromiso del Gobierno con la comunidad. El Gobierno es visto como una institución que no ofrece oportunidades de desarrollo ni garantiza el derecho a la vida y a la salud (23).

En la mayoría de los casos, al momento de estas familias acercarse a instituciones del Gobierno a solicitar ayuda, son tratadas de manera indiferente e incluso discriminatoria, lo cual genera otra forma de maltrato o victimización como consecuencia del conflicto armado interno, pero no causada por actores armados, sino por instituciones (23). La dinámica interna del conflicto arde percepciones negativas por parte de los desplazados con respecto al Gobierno, el cual es calificado como no interesado en las necesidades personales, familiares y de salud pública de la población (23).

\section{Conclusión}

La vivencia de hechos violentos conlleva fuertes impactos en la subjetividad de las personas. Por ello, la percepción que tienen las mujeres de su estado de salud-enfermedad está condicionada por factores externos a ellas, donde el desplazamiento marca un hito importante en esta significación, debido a que a partir de las consecuencias que asumen después de este hecho, se afianzan o se deterioran las prácticas culturales en el cuidado de la salud, lo cual da como resultado una nueva búsqueda del significado de bienestar, a través de la asimilación de los nuevos conceptos existentes en la ciudad a la que esta población llegan después de ser víctima de desplazamiento.

Las mujeres desplazadas construyen el concepto de salud y enfermedad a partir de las experiencias individuales de la cotidianidad, de su entorno y del contexto en el que están inmersas, donde salud y enfermedad se entienden como conceptos holísticos que trascienden lo biológico e incluyen otras dimensiones humanas como lo psicológico, las cuales se relacionan continuamente y condicionan la percepción de bienestar en sus vidas.

Sin embargo, esta percepción se ve afectada por los determinantes sociales de la salud que se encuentran luego del desplazamiento, debido a que la ciudad receptora no es garante de condiciones de vida adecuadas a las necesidades de esta población, la cual debe enfrentarse a un entorno donde priman circunstancias precarias como escasez de recursos económicos, barreras para acceder a un trabajo formal y a la educación, viviendas 
que no cumplen con requerimientos básicos de habitabilidad y carencias alimentarias y nutricionales. Estos aspectos obligan a las mujeres en situación de desplazamiento a asumir nuevos roles, a fin de favorecer el desarrollo propio y de sus familias.

Estos nuevos roles determinan la forma como las mujeres desplazadas asumen los procesos de salud-enfermedad en los diferentes entornos en los que interactúan cotidianamente, donde la familia es un factor primordial, pues siempre piensan en el bienestar de sus seres queridos y en el desarrollo de sus proyectos de vida.

Dentro de los roles que las mujeres desempeñan luego del desplazamiento está el ser las proveedoras del hogar; sin embargo, la búsqueda de un empleo que les proporcione ingresos suficientes para el sostenimiento de sus familias es compleja, por lo que la mayoría debe acceder a trabajos informales, los cuales ponen en riesgo su salud física, mental y laboral, convirtiéndolas a ellas y a sus familias en personas aún más vulnerables y reduciendo su calidad de vida. En consecuencia, se condiciona y limita la manera de afrontar los diferentes procesos de salud-enfermedad por los que se atraviesa en la cotidianidad.

La dimensión espiritual es un aspecto fundamental en las vivencias de las mujeres desplazadas, porque se convierte en una fuente de motivación y resistencia para afrontar las dificultades que se presentan. Asimismo, se apoyan en la religiosidad para encontrar un bienestar que se ve reflejado a nivel físico y mental.

Finalmente, el hecho de experimentar situaciones como la indiferencia del Estado ante sus necesidades, el desplazamiento, las condiciones de vida en la comunidad receptora y los diferentes roles que desarrollan a lo largo de su vida, influyen de alguna manera en la percepción del estado de salud-enfermedad y en los comportamientos que estas mujeres adhieren a su personalidad.

\section{Referencias bibliográficas}

1. Amnistía Internacional. Colombia: Invisibles ante la justicia. Impunidad por actos de violencia sexual cometidos en el conflicto: informe de seguimiento [Internet]. Madrid: Amnistía Internacional; 2012 [acceso: $1^{\circ}$ de septiembre del 2015]. Disponible en: http://www.acnur.org/t3/uploads/media/Colombia Invisibles_ante_la_justicia_AMR_23_031_2012. pdf? view $=1$

2. Mesa de trabajo "Mujer y conflicto armado". VI Informe sobre violencia sociopolítica contra mujeres, jóvenes y niñas en Colombia 2002-2006 [Internet]. Bogotá: Mesa de trabajo "Mujer y conflicto armado"; 2006 [acceso: $1^{\circ}$ de septiembre del 2015]. Disponible en: http://www.acnur.org/t3/ uploads/media/COI_1787.pdf?view $=1$

3. Centro Nacional de Memoria Histórica. Una nación desplazada. Informe nacional del desplazamiento forzado en Colombia [Internet]. Bogotá: Centro Nacional de Memoria Histórica; 2015 [acceso: 26 de agosto del 2016]. Disponible en: http://www.centrodememoriahistorica.gov.co/ descargas/informes2015/nacion-desplazada/unanacion-desplazada.pdf

4. Carrillo A. Buenaventura, Colombia: Realidades brutales [Internet]. Bogotá: Consejo Noruego para Refugiados; 2014 [acceso: $1^{\circ}$ de septiembre del 2015]. Disponible en: http://www.nrc.no/ arch/_img/9183876.pdf

5. Hernández A, Gutiérrez M. Vulnerabilidad y exclusión en salud: datos y relatos de la situación de la población desplazada en Bogotá [Internet]. Bogotá: Pontificia Universidad Javeriana; 2010 [acceso: 24 de agosto del 2016]. Disponible en: http://www. seminariopermanente.com/centro-de-documentacion-e-informacion/desplazamientos-forzados/ Vulnerabilidad_y_exclusion_en_salud_PROTEGIDO-1-1.pdf

6. Mogollón Pérez AS, Vázquez Navarrete ML. Opinión de las mujeres desplazadas sobre la repercusión en su salud del desplazamiento forzado. Gac Sanit. 2006 Ago.; 20(4):260-5.

7. Strauss A, Corbin J. Bases de la investigación cualitativa técnicas y procedimientos para desarrollar la teoría fundamentada. Medellín: Editorial Universidad de Antioquia; 2002. p.11-3.

8. Ortiz Giraldo A. Caracterización Comuna 3 Manrique de la Ciudad de Medellín [Internet]. Medellín: Anderson Ortiz Giraldo; 2012 [acceso: 10 de agosto del 2015]. Disponible en: http://revistacepa.weebly. com/uploads/1/3/3/7/13372958/caracterizacioncomuna3manrique.2012.pdf

9. Asociación Médica Mundial. Declaración de Helsinki. Principios éticos para las investigaciones médicas en seres humanos [Internet] [acceso: 10 
de agosto del 2015]. Disponible en: http://www.inb. unam.mx/bioetica/documentos/declaracion_helsinki.pdf

10. Colombia, Ministerio de Salud. Resolución no 008430 de 1993, por la cual se establecen las normas científicas, técnicas y administrativas para la investigación en salud. Bogotá: El Ministerio; 1993.

11. Juárez Acosta F. El concepto de salud: una explicación sobre su unicidad, multiplicidad y los modelos de salud. International Journal of Psychological Research. 2011; 4(1):70-9.

12. Organización Mundial de la Salud. Preguntas más frecuentes [Internet] [acceso: 26 de agosto del 2014]. Disponible en: http://www.who.int/suggestions/faq/es/

13. Organización Panamericana de la Salud. Conferencia Internacional sobre Atención Primaria de Salud, Alma-Ata, URSS, 6-12 de septiembre de 1978 [Internet] [acceso: 2 de septiembre del 2014]. Disponible en: http:/www1.paho.org/Spanish/DD/ PIN/alma-ata_declaracion.htm

14. Melguizo Herrera E, Álzate Posada ML. Creencias y prácticas en el cuidado de la salud. Av Enferm. 2008; XXVI(1): 112-3.

15. Berbesi Fernández DY. Alcances y limitaciones de la noción de enfermedad. CES Salud Pública. 2013; 4:47-51.

16. Vélez Arango AL. Salud y enfermedad: la contribución de la Corte Constitucional de Colombia. Hacia Promoc Salud. 2012; 17(2):91-109.

17. Ledón Llanes L. Enfermedades crónicas y vida cotidiana. Rev Cubana Salud Pública. 2011; 37(4):488-99.

18. Rodríguez Ruíz Y, Pérez Mergarejo E. Procedimiento ergonómico para la prevención de enfermedades en el contexto ocupacional. Revista Cubana de Salud Pública. 2014; 40(2):279-85.

19. Ardila Jaimes CP, Rodríguez Amaya RM. Condiciones de salud y laborales de la población trabajadora informal en situación de desplazamiento de Bucaramanga, Colombia. Investig Andina. 2013; 15(26): 628-39.
20. Marche A. Religion, health, and the care of seniors. Counselling, Psychotherapy, and Health [Internet]. 2006 [acceso: 10 de septiembre del 2014]; 2(1) 50-61. Disponible en: http://www.cphjournal.com/ archive_journals/V2_I1_Marche_50-61_04_06.pdf

21. Demera JD. Ciudad, migración y religión. Etnografía de los recursos identitarios y de la religiosidad del desplazado en Altos de Cazuca. Tehologica Xaveriana [Internet]. 2007 [acceso: 10 de septiembre del 2014]; 57(162): 303-20. Disponible en: http://www.javeriana.edu.co/Facultades/Teologia/ publicaciones/art/162(5).pdf

22. Campo A, Oviedo H, Herazo E. Prevalencia de síntomas, posibles casos y trastornos mentales en víctimas del conflicto armado interno en situación de desplazamiento en Colombia: una revisión sistemática. Rev Colomb Psiquiatr [Internet]. 2014 [acceso: 10 de septiembre del 2014]; 43(4): 1-9. Disponible en: http://www.scielo.org.co/scielo.php?script $=$ sci_ arttext\&pid $=$ S0034-74502014000400002

23. Lozano M, Gómez M. Aspectos psicológicos, sociales y jurídicos del desplazamiento forzoso en Colombia. Acta Colombiana de Psicología [Internet]. 2004 [acceso: 26 de agosto del 2016]; 12:103-19. Disponible en: http://www.redalyc.org/ articulo.oa? $\mathrm{id}=79801208$

24. García, M. Los desplazados por la violencia en Colombia. Con su dolor sin rumbo. Universitas Humanística. 1999; 47:15-31.

25. Hernández Bello A, Gutiérrez Bonilla L. Vulnerabilidad y exclusión en salud: datos y relatos de la situación de la población desplazada en Bogotá [Internet]. Bogotá: Pontificia Universidad Javeriana; 2012 [acceso: 31 de agosto del 2014]. Disponible en: http://www.javeriana.edu.co/fcea/pdfs_posg_salud/ libro_vulnerabilidad_exclusion.pdf

26. Organización Mundial de la Salud. El derecho a la salud [Internet] [acceso: 2 de septiembre del 2014]. Disponible en: http://www.ohchr.org/Documents/ Publications/Factsheet31sp.pdf 